\title{
CHAOTIC POLYNOMIALS IN SPACES OF CONTINUOUS AND DIFFERENTIABLE FUNCTIONS
}

\author{
RICHARD M. ARON \\ Department of Mathematics, Kent State University Kent, OH 44240 U.S.A. \\ e-mail: aron@math.kent.edu \\ and ALEJANDRO MIRALLES \\ Departamento de Análisis Matemático Av/ Dr. Moliner, 50 Burjassot 46100 Spain \\ e-mail: alejandro.miralles@uv.es
}

(Received 7 February, 2007; revised 12 September, 2007; accepted 14 October, 2007)

\begin{abstract}
We construct chaotic $m$-homogeneous maps acting on $\mathcal{C}_{+}^{r}([0, \infty))$ for any $m \geq 2, r \in \mathbb{N} \cup\{0\}$, and on the Fréchet spaces $\mathcal{C}_{\mathbb{R}}(\mathbb{R})$ for odd values of $m \geq 3$ and $\mathcal{C}_{\mathbb{C}}(\mathbb{R})$ for any $m \geq 2$.
\end{abstract}

2000 Mathematics Subject Classification. Primary 47A16 Secondary 47H60.

1. Introduction and background. Let $X$ be a topological space and $Q: X \longrightarrow X$ be a continuous map. We say that $x \in X$ is a periodic point if there exists $k \in \mathbb{N}$ such that $Q^{k} x=Q \underbrace{\circ \ldots \ldots \circ}_{\text {times }} Q=x$. We say that $Q$ is chaotic if

(i) $Q$ is hypercyclic; that is, there exists $y \in X$ such that $\left\{Q^{n} y\right\}_{n \in \mathbb{N}}$ is dense in $X$, and

(ii) the periodic points of $Q$ are dense in $X$.

$\mathrm{N}$. Bernardes showed in [1] that there do not exist hypercyclic homogeneous polynomials of degree $m \geq 2$ in Banach spaces. The existence of hypercyclic nonhomogeneous polynomials on Banach space was studied by A. Peris [5], who in [4] also showed that there exist chaotic homogeneous polynomials $P: \omega \longrightarrow \omega$ of degree $m \geq 2$, where $\omega=\left\{\left(z_{n}\right)_{n \in \mathbb{N}}: z_{n} \in \mathbb{C}\right\}$. Furthermore, F. Martínez [3] has studied hypercyclicity and chaos of polynomials on Köthe sequence spaces.

In the context of this paper, we will be dealing with cones and vector spaces $X$ of continuous or differentiable functions on $[0, \infty)$ and $n$-homogeneous polynomials $Q$ defined on these cones. By such a polynomial, we mean the restriction to the cone of a standard continuous $n$-homogeneous polynomial defined on the normed space generated by the cone.

We start by considering the cone

$$
\mathcal{C}_{+}([0, \infty))=\{f:[0, \infty) \longrightarrow[0, \infty): f \text { is continuous }\}
$$

with the topology of uniform convergence on compact sets, and the Fréchet spaces $\mathcal{C}_{\mathbb{R}}(\mathbb{R})$, resp. $\mathcal{C}_{\mathbb{C}}(\mathbb{R})$, of real, resp. complex, valued continuous functions on $\mathbb{R}$. We show that there exist chaotic homogeneous polynomials of degree $m \geq 2$.

We next extend our results to the topological space

$$
\mathcal{C}_{+}^{r}([0, \infty))=\left\{f:[0, \infty) \longrightarrow[0, \infty): f \in \mathcal{C}^{r}([0, \infty))\right\}
$$


endowed with the topology of uniform convergence of a function and its first $r$ derivatives on compact subsets of $[0, \infty)$.

We remark that $\omega$ is not isomorphic to $\mathcal{C}_{\mathbb{C}}(\mathbb{R})$ and therefore the spaces discussed here are new examples of spaces which admit homogeneous chaotic polynomials.

\section{Existence of hypercyclic polynomials in spaces of continuous and differentiable} functions. First we show the existence of hypercyclic polynomials in our spaces. These polynomials will be defined by $Q f(x)=f^{m}(x+\alpha)$, for some $m \in \mathbb{N}$ and some $\alpha \in \mathbb{R} \backslash\{0\}$. The idea for these polynomials comes from the fundamental work of $\mathrm{G}$. Birkhoff [2], that the operator $f(z) \rightsquigarrow f(z+\alpha)$ is hypercyclic on the space of entire functions.

We will suppose, without loss of generality, that $\alpha=1$ in the proofs. We will prove that these polynomials are actually chaotic in Section 3.

2.1. Hypercyclic polynomials on $\mathcal{C}_{+}([0, \infty))$. In this section, we first prove that $\mathcal{C}_{+}([0, \infty))$ is separable and then find hypercyclic $m$-homogeneous polynomials $Q: \mathcal{C}_{+}([0, \infty)) \longrightarrow \mathcal{C}_{+}([0, \infty))$. Note that as a subset of the separable metric space $\mathcal{C}([0, \infty)), \mathcal{C}_{+}([0, \infty))$ is automatically separable. However, we have chosen to reprove this result since the notation introduced will be useful later.

Proposition 2.1. The space $\mathcal{C}_{+}([0, \infty))$ is separable.

Proof. Let $f \in \mathcal{C}_{+}([0, \infty)), 0<\varepsilon<1$ and $J_{k}=[0, k], k \in \mathbb{N}$. By the Weierstrass approximation theorem, there is a polynomial $P \in \mathbb{Q}[x]$ such that $\|f-P\|_{J_{k}}<\varepsilon / 3$. Consider $m \in \mathbb{N}$ such that $\varepsilon / 3 \leq 1 / m \leq 2 \varepsilon / 3$. Since $f \geq 0$ everywhere, $P(x) \geq \frac{-\varepsilon}{3}$ on $J_{k}$, and so $P(x)+\frac{1}{m} \geq 0$ on $J_{k}$. Moreover it is easy that for all $x \in J_{k},\left|P(x)+\frac{1}{m}-f(x)\right|<$ $\varepsilon$. Consider the set

$$
A_{k, m}=\left\{P+1 / m: P \in \mathbb{Q}[x], P+1 / m \geq 0 \text { on } J_{k}\right\} .
$$

For each of the countably many polynomials $Q \in A_{k, m}$, we define a function $\widetilde{Q} \in \mathcal{C}_{+}([0, \infty])$ by $\widetilde{Q}(x)=Q(x)$ if $x \in J_{k}$ and $\widetilde{Q}(x)=Q(k)$ if $x \geq k$.

Then $\widetilde{A}=\bigcup_{k, m \in \mathbb{N}}\left\{\widetilde{Q}: Q \in A_{k, m}\right\}$ is our countable dense set in $\mathcal{C}_{+}([0, \infty))$.

In order to present our example of a hypercyclic polynomial on $\mathcal{C}_{+}([0, \infty))$, the following notation will be helpful. For $k \in \mathbb{N}, k$ being even, and for $n \in \mathbb{N}, 1 \leq n \leq k$, we let $I_{n, k}=[a(n, k), b(n, k)]$ be an interval of length $k$ having positive integer endpoints, chosen so that the distance between any two such intervals is at least 1 .

TheOREM 2.2. The map $Q: \mathcal{C}_{+}([0, \infty)) \longrightarrow \mathcal{C}_{+}([0, \infty))$ defined by $Q f(x)=f^{m}(x+$ $\alpha)$ is hypercyclic for any $m \in \mathbb{N}$ and $\alpha \in \mathbb{R}, \alpha>0$.

Proof. Fix $m \in \mathbb{N}$ and $\alpha=1$. Let $\left\{P_{1}, P_{2}, \ldots\right\}$ be an enumeration of the countable set $\widetilde{A}$ described in Proposition 2.1 and define $f$ on $\bigcup_{1 \leq n \leq k, k \text { even }} I_{n, k}$ by

$$
f(x)=\sqrt[m^{a(n, k)}]{P_{n}(x-a(n, k))}
$$

for $x \in I_{n, k}, k \in \mathbb{N}, k \geq 0$ and $1 \leq n \leq k$. We extend $f$ linearly between the intervals $I_{n, k}$ and obtain that the function $f$ is non-negative and continuous on $\mathbb{R}$. In order to prove that $\left\{Q^{m} f\right\}_{m \in \mathbb{N} \cup\{0\}}$ is dense in our space, it is enough to consider functions in $\widetilde{A}$. Let $P_{n} \in \widetilde{A}, \varepsilon>0$ and $K=[0, k]$ for any $k \in \mathbb{N}$. Let $g:[0, \infty) \longrightarrow[0, \infty)$ be the continuous 
function defined by $g(x)=f^{m^{a(n, k)}}(x+a(n, k))=Q^{a(n, k)} f(x)$. Then for $x \in K$ we have that $\left|g(x)-P_{n}(x)\right|=\left|P_{n}(x)-P_{n}(x)\right|=0$, which implies that $\left\|Q^{a(n, k)} f-P_{n}\right\|_{K}<\varepsilon$ and therefore $Q$ is hypercyclic.

2.2. Hypercyclic polynomials on spaces of continuous and differentiable functions. We begin with the continuous case, showing that there exist hypercyclic homogeneous polynomials acting on $\mathcal{C}_{\mathbb{R}}(\mathbb{R})$ and $\mathcal{C}_{\mathbb{C}}(\mathbb{R})$. Later we will show that they are actually chaotic. For our argument, it will be helpful to let $c(n, k)=(a(n, k)+b(n, k)) / 2$.

THeOREM 2.3. The polynomial $Q: \mathcal{C}_{\mathbb{R}}(\mathbb{R}) \longrightarrow \mathcal{C}_{\mathbb{R}}(\mathbb{R})$ defined by $Q f(x)=f^{m}(x+\alpha)$ is hypercyclic in $\mathcal{C}_{\mathbb{R}}(\mathbb{R})$ for odd values of $m \in \mathbb{N}$ and $\alpha \in \mathbb{R} \backslash\{0\}$.

Proof. Let $m \in \mathbb{N}$ be an odd number. Let $\left\{P_{n}\right\}_{n \in \mathbb{N}}$ be an enumeration of the countable dense set $\mathbb{Q}[x] \subset \mathcal{C}_{\mathbb{R}}(\mathbb{R})$. We define

$$
f(x)=\sqrt[m^{c(n, k)}]{P_{n}(x-c(n, k))}
$$

for every $x \in I_{n, k}, 1 \leq n \leq k+1$, and we extend continuously to $\mathbb{R}$. Note that $m^{c(n, k)}$ is an odd integer, and therefore $f$ is continuous on $\mathbb{R}$.

Take arbitrary $P_{n} \in \mathbb{Q}[x], \varepsilon>0$ and $K=[-k / 2, k / 2]$. It is clear that $x+c(n, k) \in$ $I_{n, k}$ for any $x \in K$. Then, if $x \in K$ we have

$$
Q^{c(n, k)} f(x)=f^{m^{(n, k)}}(x+c(n, k))=P_{n}(x)
$$

so $\left|Q^{c(n, k)} f(x)-P_{n}(x)\right|=\left|P_{n}(x)-P_{n}(x)\right|=0<\varepsilon$ and $Q$ is hypercyclic.

In the rest of this section, we consider two extensions of the above theorem. We first look at complex-valued functions. A similar argument shows the following theorem:

THEOREM 2.4. The polynomial $Q: \mathcal{C}_{\mathbb{C}}(\mathbb{R}) \longrightarrow \mathcal{C}_{\mathbb{C}}(\mathbb{R})$ defined by $Q f(x)=f^{m}(x+\alpha)$ is hypercyclic for any $m \in \mathbb{N}$ and $\alpha \in \mathbb{R} \backslash\{0\}$.

In this situation, although there is no longer any need to restrict to odd integers $m$, it is necessary to explain why one can take roots of $\mathbb{C}$-valued functions. To see this, notice that for $x \in I_{n, k}, P_{n}(x-c(n, k))$ can be written as $\rho(x) e^{i \beta(x)}$, where $\rho \geq 0$ and $\beta$ are continuous. Thus, on $I_{n, k}, f(x)=\sqrt[m^{\alpha(n, k)}]{\rho(x)} \exp \left(i \beta(x) / m^{\alpha(n, k)}\right)$ is continuous.

We turn next to the differentiable case. Let $\left\{P_{j}\right\}$ be a countable set of $\mathcal{C}^{\infty}$ functions on $[0, \infty)$ such that $P_{j}(x)>0$ for every $j \in \mathbb{N}$ and every $x \geq 0$. It is not difficult to show that the function given by $f(x)=\sqrt[m(n, k)]{P_{n}(x-c(n, k))}$ for $x \in I_{n, k}$ can be extended to be in $\mathcal{C}^{\infty}([0, \infty))$. Arguing as in Theorem 2.3, we have the following result.

THEOREM 2.5. Let $r \in \mathbb{N} \cup\{\infty\}$. The m-homogeneous polynomial $Q$ : $\mathcal{C}_{+}^{r}([0,+\infty)) \longrightarrow \mathcal{C}_{+}^{r}([0,+\infty))$ defined by $Q f(x)=f^{m}(x+\alpha)$ is hypercyclic for any $m \in \mathbb{N}$ and $\alpha \in \mathbb{R} \backslash\{0\}$.

3. Chaotic polynomials in spaces of continuous and differentiable functions. Firstly, we prove that the polynomials found in Theorem 2.4 are actually chaotic for $\mathcal{C}_{\mathbb{C}}(\mathbb{R})$.

THEOREM 3.1. Let $Q: \mathcal{C}_{\mathbb{C}}(\mathbb{R}) \longrightarrow \mathcal{C}_{\mathbb{C}}(\mathbb{R})$ be the $m$-homogeneous polynomial given by $Q f(x)=f^{m}(x+\alpha)$. Then $Q$ is chaotic for any $m \in \mathbb{N}$ and $\alpha \in \mathbb{R} \backslash\{0\}$. 
Proof. We show that there exists a dense subset of $\mathcal{C}_{\mathbb{C}}(\mathbb{R})$ whose elements are periodic, from which it will follow that the polynomial $Q$ is chaotic. For any $P \in$ $\mathbb{Q}[x]+i \mathbb{Q}[x]$ and $k \in \mathbb{N}$ we argue as indicated for Theorem 2.4 above, constructing a continuous function $P_{k}: \mathbb{R} \longrightarrow \mathbb{C}$ defined first on $[-k, k+1)$ by

$$
P_{k}(x)=\left\{\begin{aligned}
P(x) & \text { if } x \in[-k, k] \\
\sqrt[m^{2 k+1}]{P(-k)} & \text { if } x=k+1,
\end{aligned}\right.
$$

extending continuously in $(k, k+1)$. Note that

$$
Q^{2 k+1} P_{k}(-k)=P_{k}^{m^{2 k+1}}(k+1)=P(-k)=P_{k}(-k) .
$$

Put $x_{p}^{k}=(2 k+1) p-k$ for any $p \in \mathbb{Z}$ and define

$$
P_{k}(x)=\sqrt[m^{p(2 k+1)}]{P_{k}(x-p(2 k+1))} \text { for } x \in\left[x_{p}^{k}, x_{p+1}^{k}\right) .
$$

Now, $P_{k}$ is continuous on the open intervals $\left(x_{p}^{k}, x_{p+1}^{k}\right)$, and $\lim _{x \rightarrow x_{p+1}^{k}} P_{k}(x)=$ $\lim _{x \rightarrow x_{p+1}^{k}} P_{k}(x)=\sqrt[m(p+1)(2 k+1)]{P(-k)}=P_{k}\left(x_{p+1}^{k}\right)$.

The limit from the left is

$$
\begin{aligned}
\lim _{x \rightarrow x_{p+1}^{k}-} P_{k}(x) & =\sqrt[m^{p(2 k+1)}]{P_{k}((2 k+1)(p+1)-k-p(2 k+1))} \\
& =\sqrt[m^{p(2 k+1)}]{P_{k}(k+1)} \\
& =\sqrt[m^{p(2 k+1)}]{m^{2 k+1} \sqrt{P(-k)}} \\
& =\sqrt[m^{(p+1)(2 k+1)}]{P(-k)}
\end{aligned}
$$

and the limit from the right is

$$
\begin{aligned}
\lim _{x \rightarrow x_{p+1}^{k}+} P_{k}(x) & =\sqrt[m^{(p+1)(2 k+1)}]{P_{k}((2 k+1)(p+1)-k-(p+1)(2 k+1))} \\
& =\sqrt[m^{(p+1)(2 k+1)}]{P(-k)} .
\end{aligned}
$$

As a consequence, the function is continuous at $x_{p}^{k}$ for every $p \in \mathbb{Z}$, and thus $P_{k}$ is continuous on $\mathbb{R}$. $P_{k}$ is also $Q$-periodic since $Q^{2 k+1} P_{k}(x)=P_{k}(x)$ for all $x \in \mathbb{R}$. Consider the countable set

$$
D=\left\{P_{k}: P \in \mathbb{Q}[x]+i \mathbb{Q}[x], k \in \mathbb{N}\right\} .
$$

We have that every function in $D$ is periodic for $Q$, and dense in $\mathcal{C}_{\mathbb{C}}(\mathbb{R})$. Indeed, let $f$ be a continuous function $f: \mathbb{R} \longrightarrow \mathbb{C}, \varepsilon>0$ and $K=[-k, k], k \in \mathbb{N}$. There is $P \in \mathbb{Q}[x]+i \mathbb{Q}[x]$ such that $\|f-P\|_{K}<\varepsilon$. Then we have that $\left\|f-P_{k}\right\|_{K}<\varepsilon$ and $Q$ is chaotic.

REMARK 3.2. By Theorem 3.1, the maps $Q$ found in our spaces $\mathcal{C}_{+}([0, \infty))$ and $\mathcal{C}_{\mathbb{R}}(\mathbb{R})$ are also chaotic. The fact that these polynomials are chaotic in $\mathcal{C}_{+}^{r}([0, \infty))$ is clear since $\widetilde{A}$ is a dense subset in this space and we can keep the differentiability in the proof of the Theorem 3.1. 
ACKNOWLEDGEMENT. The authors are grateful to the referee whose many helpful suggestions resulted in a much improved manuscript.

\section{REFERENCES}

1. N. Bernardes. On orbits of polynomial maps in Banach spaces, South African Math. Soc. 21 (1998), 311-318.

2. G. Birkhoff, Démonstration d'un théoréme elémentaire sur les fonctions entiéres, $C . R$. Acad. Sci. Paris 189 (1929), 473-475.

3. F. Martínez Giménez, Universalidad, hiperciclicidad, y caos en espacios de Fréchet, Thesis (Universidad Politécnica de Valencia 2000).

4. A. Peris, Chaotic polynomials on fréchet spaces, Proc. Amer. Math. Soc. 127 (1999), 3601-3603; Erratum to Chaotic polynomials on fréchet spaces, Proc. Amer. Math. Soc. 129 (2001), 3759-3760.

5. A. Peris, Chaotic polynomials on banach spaces, J. Math. Anal. Appl. 287 (2003), 487-493. 